\title{
Dynamic Idle Mode Control in Small Cell Networks
}

\author{
Bahar Partov $^{1}$, Douglas J. Leith ${ }^{1}$, Rouzbeh Razavi ${ }^{2}$, and Holger Claussen ${ }^{2}$ \\ ${ }^{1}$ Hamilton Institute, NUI Maynooth, ${ }^{2}$ Bell Laboratories, Alcatel-Lucent, Dublin
}

\begin{abstract}
The deployment of both outdoor and indoor Small Cell Base Stations (SCBSs) has attracted significant interest in the wireless industry. However, a critical concern in a large scale deployment is the efficient control of the small cell's transmission mode. In this paper we propose a load aware approach for dynamic idle mode selection where the load distribution is estimated by the "RF Fingerprints" of the users. Our approach allows pilot signals and most of the processing power of a SCBS to be completely switched off when no active user is in its vicinity, or when the required Quality of Service (QoS) can be provided by the underlying macro base stations. Such an approach significantly reduces the energy consumption of the small cells as well as reducing the pilot pollution and signalling overhead. We evaluate the efficiency of our approach using field measurements in central Dublin as well as with system-level simulations. We show that the proposed method is capable of identifying idle cells with an average prediction error rate of $1.9 \%$. Moreover we show that it has the potential to achieve an average reduction of $90 \%$ in $\mathrm{kWh}$ power consumption compared to the concurrent operation of all SCBSs.
\end{abstract}

Index Terms-Small cells, Idle Mode Control, Energy Efficiency

\section{INTRODUCTION}

In this paper we propose a new method for idle mode control of small cells. Our approach differs from the existing work in that we make use of the available measurements of the received signal powers ("RF Fingerprints") from the underlying macro BSs reported by the User Equipments (UEs). By using a central database of RF Fingerprints we show that, even without transmission of the pilot powers by the small cells, when an appropriate classifier is used, desirable cell associations can be determined with an average confidence rate of $98.1 \%$. Moreover we show that by applying our proposed method a significant reduction in power consumption of small cells (on the order of $90 \%$ ) can be achieved compared to full power operation of all the small cells and savings of $58 \%$ compared to existing idle mode control procedures.

It is predicted that global mobile data traffic will increase by 10-fold between 2014 and 2019 [1]. In order to meet this traffic demand, the cellular network architecture is shifting towards the use of a heterogeneous approach comprising a mix of macro and small cells. The number of deployed small cells is forecast to increase from 2.3 million at the end of 2010 to 54 million by 2015 [2]. In contrast to macro BS deployment, SCBSs are deployed with a higher degree of randomness since they can be installed by the end user or in an opportunistic manner. Consequently in a large scale

This material is based upon works supported by the Science Foundation Ireland under Grant No. 11/PI/1177 and by Bell Labs Ireland. deployment, it is increasingly critical to address the following issues:

- Energy Saving: It is well established that offloading the macro base station load can potentially yield significant energy savings [3]. However the deployment of millions of small cells raises concerns about the aggregate power consumption of those BSs. For example, an average of $12 \mathrm{~W}$ operation power for each small cell equates to power consumption of $105.12 \mathrm{kWh}$ per year; resulting in a consumption of $5.25 \times 10^{9} \mathrm{kWh}$ per year for 50 million small cells. The $\mathrm{CO}_{2}$ emissions of dense deployment can also become significant depending on the type of power source used. Further, provisioning of power and backhaul for dense deployments is in itself a major challenge for network operators [4]. A potential solution is to use wireless back-haul and alternative energy sources, e.g. solar energy [5], in which case it is essential for small cells to efficiently manage their power consumption. While in sleep mode small cells have reduced power usage, depending on the hardware architecture and sleep mode algorithms [6] the reduction is typically limited to no more than $30 \%$ to $60 \%$ of the total power consumption. To achieve the largest energy savings, it is necessary to place the small cell base stations into deep sleep mode where the energy consumption is essentially zero.

- Interference and Pilot Pollution: SCBSs can cause interference to the users of the macro BSs as well as interference to the users of the other SCBSs, resulting in undesirable impacts on the performance. Moreover denser small cells result in pilot pollution where multiple strong transmitters are detected by a UE. Enhanced Inter Cell Interference Coordination (eICIC) methods are developed in releases 10 and 11 of 3GPP [7] to enhance interoperability between the layers. Cell Range Expansion (CRE) allows more effective cell selection methods, where offset values are added to the small cells received powers to increase their coverage range [8]. Almost Blank Subframes (ABS) mitigate interference between macro BS and SCBS by muting macro transmission at specific subframes. Putting SCBSs into sleep mode when no pilot power is transmitted, potentially provides an additional means of interference management.

- Signalling Overhead: In busy areas significant signalling overhead can be introduced by the handover/hand-off messages of passers-by in the vicinity of a small cell. Further enhancements in release 12 of the LTE specification address these problems. For example dual connectivity modes allow simultaneous connection of the user to both 
macro and small cell layers, enabling separation of the data and control messages, where the control signalling is provided by the macro layer and high speed data is provided by the small cell layer. Once again, SCBS idle mode control potentially provides an additional mechanism to manage signalling overhead.

Although each of the issues above can and must be addressed separately, it should be noted that the traffic load demand plays a critical role in defining dynamic configurations of the network. A load aware selection of the small cells adjusts the number of active cells based on the traffic load demand. Due to the dynamic behaviour of the traffic demand, this approach inherently reduces the levels of pilot pollution and signalling overhead as well as introducing energy efficiency gains.

Idle mode control procedures for small cells have received relatively little attention in the literature to date. In [6] the authors propose sleep mode algorithms by enabling dynamic adjustment of the small cell transmit powers. In our previous work [9], we introduced deep sleep mode schedules as well as independent adjustment of the pilot and data powers, where we made use of long term characteristics of the traffic demand. In [10], the authors propose distributed procedures for idle mode control, where each small cell base station remains in idle mode unless it detects user activity in its vicinity. A small cell determines user activity by sniffing the uplink channel between a UE and its serving macro BS. Multiple wake ups, pilot pollution and plug-in power consumption are potential disadvantages of these distributed methods. In release 11 of the 3GPP [11], a number of waking up procedures are proposed for hotspot cells. We complement these wakeup based approaches by reducing pilot transmissions; to estimate the topology of the network load and to control idle modes accordingly, we make use of information on the received power vectors or the "RF Fingerprints" reported by the UE.

The rest of the paper is organized as follows. In Section II we introduce our approach towards using the RF Fingerprints. In Section III we introduce our proposed classification method. In Section IV we carry out a performance evaluation of field data in central Dublin as well as a performance evaluation of the synthetic data which are generated by system-level simulations. And finally, in Section V, we summarise our conclusions.

\section{RF FINGERPRINTS AND IDLE MOdE SELECTION}

Let $\mathcal{B}$ denote the set of base stations, where $\mathcal{B}_{m} \subset \mathcal{B}$ is the set of macro BSs, and $\mathcal{B}_{s} \subset \mathcal{B}$ is the set of SCBSs. We denote the set of users in the geographic area of $\mathcal{B}$ by $\mathcal{U} . p_{b}^{p}$ and $p_{b}$ denote the pilot and transmit powers of BS $b$. When all small cell base stations are active, a user is scheduled to a base station according to one of the following rules:

- Signal Strength: Maximum received pilot power:

$$
b_{u} \in \underset{i \in \mathcal{B}}{\arg \max } p_{i}^{p} h_{i, u}
$$

- Signal Quality: Maximum pilot SINR:

$$
b_{u} \in \underset{i \in \mathcal{B}}{\arg \max } \gamma_{b, u}
$$

where

$$
\gamma_{b, u}=\frac{p_{i}^{p} h_{i, u}}{\sigma^{2}+\sum_{k \in \mathcal{B} \backslash\{b\}} p_{k} h_{k, u}}
$$

with $b_{u}, h_{b, u}$ and $\sigma^{2}$ denoting the candidate serving cell for user $u$, the channel gain between BS $b$-user $u$ and the noise power respectively. These cell selection rules require all of the base stations to be active and so result in increased power consumption as well as excessive interference and signalling overhead.

We denote the received pilot powers from nearby macro BSs as the RF Fingerprint of a user. Here nearby refers to BSs for which received pilot power is above the detection level of the UE. In our approach instead of using the received pilot powers for $\forall b_{i} \in \mathcal{B}, i=1, . .,|\mathcal{B}|$, we make use of an existing database of RF fingerprints to estimate the serving cell for each user. This database is built in a central network entity, based on the past records of the RF fingerprints from $\forall b_{i} \in \mathcal{B}_{m}, i=$ $1, . .,\left|\mathcal{B}_{m}\right|$. We denote UE $u$ 's RF fingerprint vector by $X_{u}$, where each vector $X_{u}$ is associated with a class label, namely UE's serving BS, $b_{u}$. For a given RF Fingerprint vector $X_{u}, b_{u}$ can be calculated using Eq 2. A training database $\mathcal{T}$, therefore contains a set of RF Fingerprint vectors $X_{u}$ together with their corresponding class label $b_{u}$. The task of the classifier $\mathcal{C}$, is to use the training data $\mathcal{T}$, to predict cell association for a query fingerprint vector, $Y_{u^{\prime}}$.

As a routine part of their operation, UEs periodically send measurement reports of the received pilot powers. In addition, a BS can explicitly request UEs to perform and report measurements for making handover decisions. Hence collecting RF-fingerprints does not introduce additional overhead or operational cost.

\section{CLASSIFICATION OF THE RF FINGERPRINTS}

\section{A. Classification Criteria}

We use a cell association rule based on pilot signal quality introduced in Section II. As previously stated simultaneous operation of densely deployed SCBSs can cause pilot pollution and consequently impact the user's experience. Hence, we consider sets of training data for which RF fingerprint vectors are governed by the received pilot powers from the macro BS network and when network of the small cells is deactivated. However to determine the class label i.e. the serving cell for each RF Fingerprint vector, we need to calculate the signal quality from both macro BS and SCBS networks. The received pilot power for each UE $u$ from BS $b \in \mathcal{B}_{m}$ can be driven from UE's RF fingerprint vector. To estimate the achievable signal qualities from the small cells, we use standard urban path loss models [12] where the received pilot powers from $b \in \mathcal{B}_{s}$ are calculated based on the path loss model. When calculating the signal quality, we consider use of blank subframes by the macro cells so that interference from the macro to neighbour small cells is minimal.

\section{B. Data Matching and Similarity Metrics}

In practice fingerprint vectors could contain missing entries. Considering the incompleteness of the fingerprint vectors, 
fitting probability distributions to the observation data is likely to be inaccurate. Hence we consider use of deterministic Knearest neighbour classifiers. K-nearest methods are computationally simple which also makes them suitable for dynamic updates. A typical nearest neighbour method uses the pairwise Euclidean distance to measure the similarities. To reduce sensitivity to abrupt changes in the Received Signal Code Power (RSCP) values, we instead use the 1 norm distance. A KNN classifier with the 1 norm similarity measure could identify two geographically distant observations as similar e.g.

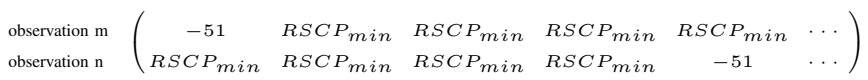

where $m$ and $n$ are observations at two distant locations and $R S C P_{\text {min }}:=\min _{u \in \mathcal{U}}\left\{\min _{i \in X_{u}} X_{u, i}\right\}$ is a substitute for missing entries i.e. very low received powers which are not detectable by the UE. To avoid possible errors of this type, we construct definition of sets of serving/neighbouring BSs. Each observation or Fingerprint vector in fact relates to a set of base stations which are detectable by the UE. Let $\mathcal{S}_{i}$ be the corresponding neighbouring set to the fingerprint vector $X_{i}$, we first find $N_{J}$ most similar observations to the test data in terms of their neighbouring BS sets. Then we apply a 1 norm K-Nearest Neighbour search on the remaining $N_{J}$ observations. By using this method we practically filter-out observation which can cause prediction errors. To determine the similarity measures between different neighbouring sets we make use of the "Jaccard" similarity index. The proposed two stage classifier hence comprises the following steps:

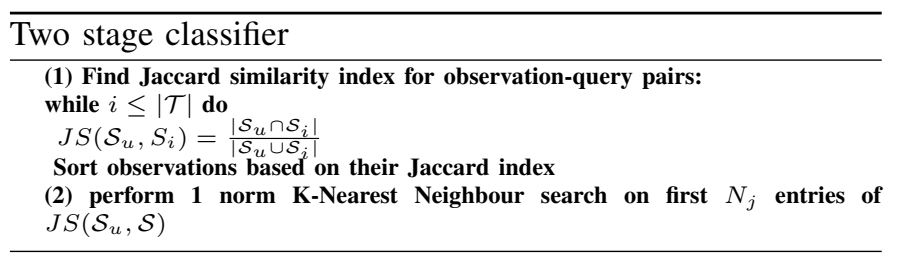

Upon finding the $\mathrm{K}$-nearest observations, the test vector is classified by majority vote of the $K$ neighbours. It is also common to apply weights to the votes to reduce error. In this work we consider weights proportional to the inverse of the 1 norm distance for each test-observation pair.

\section{Practical Implications}

Table I illustrates a sub-sample of the fingerprint vectors based on field measurements in central Dublin. Classification based on the actual measurements of the UEs could be challenging for the following reasons:

- Measurements of the RSCPs by the UEs could be noisy due to the characteristics of the wireless medium. Moreover, there exists some uncertainty in the measurement capability of the device.

- Fingerprint vectors $X$ contain a number of empty elements. Besides, the probability of having sparse vectors increases in a LTE network where soft handover is not implemented.
TABLE I: Sub-samples of RF fingerprint pilot powers in $\mathrm{dBm}$ where each dimension is marked by a distinct Primary Scrambling Code, PSC and each distinct measurement point is identified by a geographical coordinates, $l$.

\begin{tabular}{|l||c|c|c|c|c|c|c|c|}
\hline Location PSCs & 212 & 204 & 252 & 300 & 120 & 45 & 236 & 292 \\
\hline \hline$l_{1}:(53.3400988,-6.2607508)$ & - & -69 & -53 & - & -105 & -91 & -68 & -65 \\
\hline$l_{2}:(53.3401079,-6.2607396)$ & - & - & - & - & - & - & - & -63 \\
\hline$l_{3}:(53.3401169,-6.2607290)$ & - & - & - & - & - & - & - & -63 \\
\hline$l_{4}:(53.3401227,-6.2607128)$ & - & - & -51 & - & - & - & - & - \\
\hline$l_{5}:(53.3401297,-6.2607026)$ & - & - & -51 & - & - & - & - & - \\
\hline$l_{6}:(53.340137,-6.2606895)$ & - & - & -51 & - & - & - & -60 & -59 \\
\hline
\end{tabular}

To avoid erroneous measurements by the UE at a single location, measurements could be repeated and compared against each other so that a more reliable fingerprint vector per location can be derived. Furthermore, due to the dynamic characteristics of the radio environment, measurements can be re-evaluated at different time intervals, and data sets can be updated accordingly. Once the randomness of the measured signal strengths is roughly smoothed out by multiple measurements, empty entries are indicative of very low RSCP values.

\section{Performance Evaluation}

For evaluation, we collected field measurements in central Dublin outdoor locations in June 2014. We equipped an Android mobile device with a major network provider's 3G sim card and collected the measurements by war-driving. We modified an open source Android App [13] to collect signal strength measurements from neighbouring cells in addition to collecting the geographical coordinates. We only use geographical coordinates for validation purposes. Figure 1a shows a sample path where the measurements were taken. Measurements were taken with an average walking speed of $3 \mathrm{Km} / \mathrm{h}$ and at $1 \mathrm{~s}$ intervals.

\section{A. Cross Validation and Misclassification Error}

We first consider a scenario where SCBSs are placed deterministically in hotspot locations as shown in Fig 1b. We use a 3GPP path loss model to estimate the received powers and SINR values from the SCBSs. SCBS parameters can be found in Table II. We consider following three classifiers:

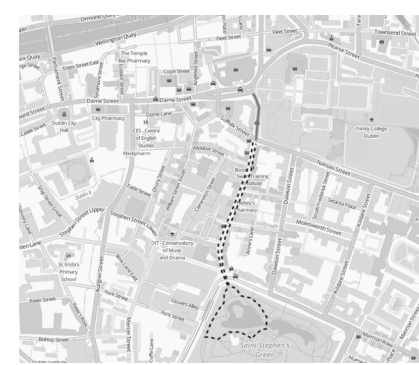

(a)

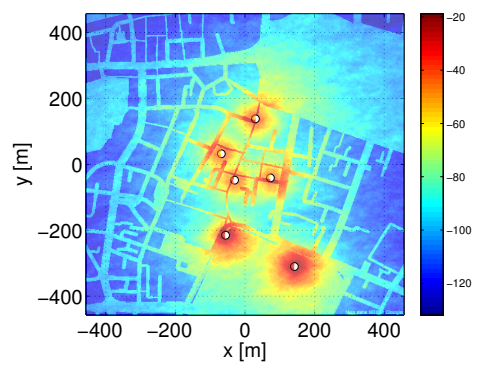

(b)
Fig. 1: (a): Fingerprint measurement path- (b): Received power map from the SCBSs. 
- (a) Classifier which performs 1 norm KNN search through the observations in the training set with common neighbouring cells i.e. $\left\{X_{i} \in \mathcal{T} \mid \mathcal{S}_{u} \cap \mathcal{S}_{i} \neq \emptyset\right\}$.

- (b) Classifier which performs 1 norm KNN search on the entire training set.

- (c) Two stage classifier proposed in Section III.

To determine the misclassification error, we apply a 10-fold cross validation to the entire measurement set within the walking path. The misclassification error is defined as follows. Let $\hat{\mathcal{C}}(X)$ and $\boldsymbol{b}$ denote the predicted and target cell association vectors respectively. A loss function is defined as a mismatch between the classifier's predictions and the target values:

$$
L\left(\boldsymbol{b}, \hat{\mathcal{C}}\left(X_{u}\right)\right)=I(\boldsymbol{b} \neq \hat{\mathcal{C}}(X))
$$

where $\mathrm{I}$ is the indicator function. Hence the generalisation error can be defined as the prediction error over an independent test sample:

$$
E r r_{\mathcal{T}}=\mathbb{E}_{X}[L(\boldsymbol{b}, \hat{\mathcal{C}}(X)) \mid \mathcal{T}]
$$

Expected prediction error, on the other hand averages over everything that is random including the randomness in the training set that produced $\hat{\mathcal{C}}[14]$ :

$$
\operatorname{Err}=\mathbb{E}[L(\boldsymbol{b}, \hat{\mathcal{C}}(X))]=\mathbb{E}\left[E r r_{\mathcal{T}}\right]
$$

Here we referred to $E r r_{\mathcal{T}}$ as the misclassification error of classifier trained on $\mathcal{T}$, and $\operatorname{Err}$ as the expected misclassification error. Figure 2 shows the misclassification error of the three

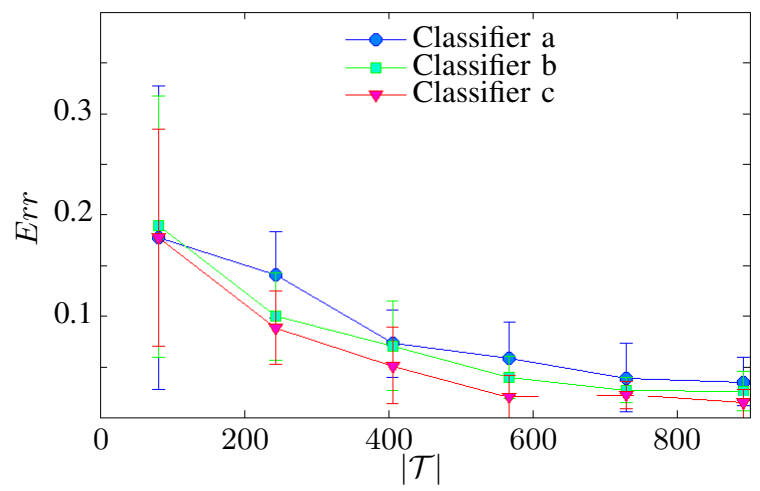

Fig. 2: Misclassification error when 1st nearest neighbour is used with classifiers (a), (b), and (c) and versus size of the training data, $|\mathcal{T}|$.

classifiers when a 1 st nearest neighbour search is used. It can be seen that a misclassification error of $1.9 \%$ is achievable using classifier (c) and the complete data set. Figure 2 also indicates that the performance improves for all classifiers with increasing size of the training data. The proposed two stage classifier slightly enhances the performance compared to the other classifiers. As the size of the training data decreases, all classifiers perform similarly. This is related to the correlation of the fingerprint samples: measurement records suggest that there can be abrupt changes in fingerprint vectors which are a few tens of meters apart. A decrease in the size of the training data implies more dissimilarities between the training set and

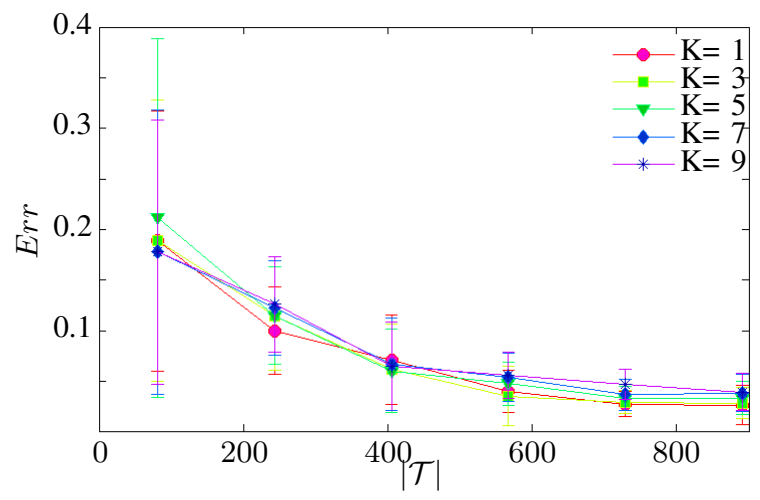

Fig. 3: Misclassification error of the classifier (b) with KNN versus size of the training data, $|\mathcal{T}|$.

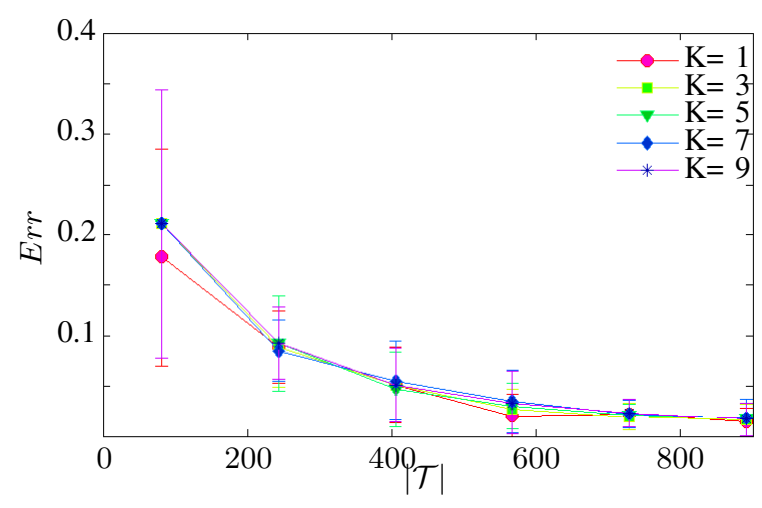

Fig. 4: Misclassification error of the classifier (c) when KNN used at the second stage, versus size of the training data, $|\mathcal{T}|$.

validation data and hence a larger mean and variance in the prediction error. Figures 3 and 4 show the misclassification error versus size of the training data for classifiers (b) and (c) respectively and when number of nearest neighbours is varied from 1 to 9 . It can be seen that increasing the number of neighbours does not necessarily improve the performance of the classifier. Moreover with decreasing size of the training data, the 1-nearest neighbours method outperforms the Knearest methods for the same reason of decreased similarity among observations and test data.

To validate the classification error under various configurations, SCBSs are evenly located across the validation points. Figure 5 shows the misclassification error for a varying number of SCBSs and with a complete training set. Each box plot represents the prediction error for $M$ SCBSs. The boxes have lines at the lower quartile, median, and upper quartile values. It can be observed that the misclassification error is increasing with increasing density of the SCBSs. As the density of the small cells is increasing, more validation points can lie at the edges of the cells resulting in increased probability of misclassification. The observed errors however can be split into three categories:

- User should be served by MBS but it is classified as being served by a SCBS.

- User should be served by SCBS but it is classified as 


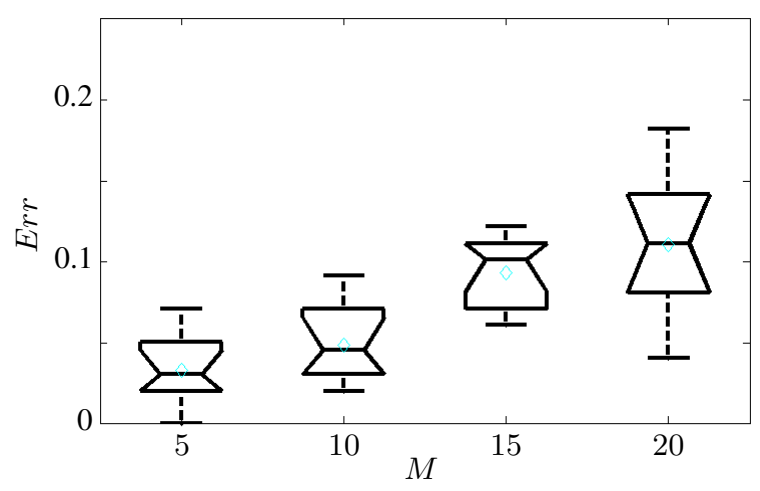

Fig. 5: Misclassification error with Classifier (c)/1st nearest neighbour and complete training set versus number of SCBSs, $M$.

being served by a MBS

- User association is misclassified between different SCBSs.

The type of misclassification error depends on the configuration of the network i.e. density of the small cells and their relative positions to the macro BSs. For example when small cells are located closer to the centre of a macro BS, the misclassification probability between macro and micro users increases. To assess the different types of misclassification error only based on the density of the SCBSs, we consider evenly positioned SCBSs across the walking path. To vary the density of the BSs we used various inter-hotspot distances. Figure 6 illustrates the split misclassification errors for such placement of SCBSs.

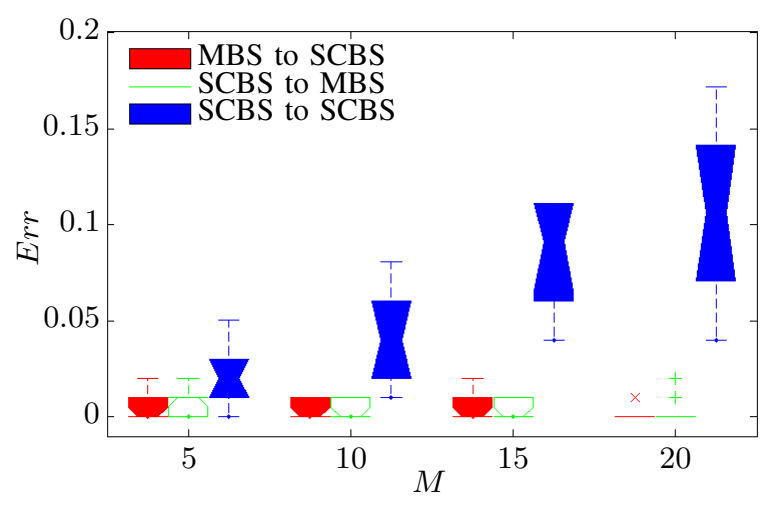

Fig. 6: Different types of misclassification with Classifier (c)/1st nearest neighbour versus number of SCBSs, $M$.

Irrespective of the type of the misclassification error, the associated fingerprint vector could fall into one of the following categories:

- Fingerprint vectors with single entries i.e. when no neighbouring cell has been detected. These errors account for the $7.6 \%$ of the total observations. As well as accounting for an average of $40 \%$ of the error when classifier (b) is used.
- Fingerprint vectors where the signal from the serving cell is suddenly lost due to obstructions (shops, etc,..). This is when user is handed over to a base station which is not in the current list of neighbours.

\section{B. Evaluation Using Synthetic Data Records}

1) Misclassification Error and Network Configuration: To further assess the potential effects of the network configuration on the misclassification error, we consider a set of simulations with a given location of the MBSs in central Dublin [15]. To simulate different configurations of the SCBSs, we consider circles of radius $\mathrm{R}$ centred at each macro $\mathrm{BS}$ location. We place 4 SCBSs on the circumference of each circle and we change the radius $\mathrm{R}$ to generate various topologies of the SCBS's locations. We consider an outdoor simulation area of $300 \mathrm{~m} \times$ $300 \mathrm{~m}$. Figures 7 and 8 illustrate the locations of SCBSs and macro BSs together with the estimated serving cell maps. And table II summarises the simulation parameters.

TABLE II: Simulation parameters

\begin{tabular}{|l|c||c|r|}
\hline Parameter & Value & Parameter & Value \\
\hline MBS Tx Power & $46 d B m$ & SCBS Tx Power & $30 d B m$ \\
\hline MBS Ant. Height & $25 m$ & SCBS Ant. Height & $10 \mathrm{~m}$ \\
\hline MBS Ant. Gain & $15 d B i$ & SCBS Ant. Gain/Type & $2.15 d B i /$ Dipole \\
\hline MBS Ant Tilt & $10^{\circ}$ & UE Ant. Height & $1.5 \mathrm{~m}$ \\
\hline UE Ant. Gain/Type & $0 d B i / O m n i$ & UE Noise Figure & $9 d B$ \\
\hline Path Loss model & {$[12]$} & Shadow fading STD & $6 d B$ \\
\hline
\end{tabular}

Here we refer to the synthetic data as the RF fingerprint vectors generated by simulations and by using urban path loss models. Since these fingerprint vectors do not contain empty entries, classifier (b) is used. Figure 9 illustrates the misclassification error types for the various network configurations. Unlike the results in Fig 6, the percentage of misclassification of an estimated SCBS with another SCBS is relatively low. This is due to the different network topology and the position of the SCBSs. As the SCBSs in the previous Section were positioned in close distance, it's more likely to incorrectly classify among them. While Figs 7 and 8 indicate larger coverage area by the macro BSs identified by Cell ID 1; resulting in higher misclassification error between MBS and SCBS pairs.

2) Energy Efficiency Gains: Gains in energy efficiency are a natural by-product of the proposed idle-mode control procedure. To evaluate potential energy saving gains the spatiotemporal characteristics of the user traffic demand must be considered. We consider the network configurations described in the previous Section. Figure 10 and Table III summarise the behaviour of the normalised traffic load within the simulation area for various hours of the day and traffic demands parameters respectively. To simulate temporal and spatial variations of the load, we divide 24 hours into 5 minute snapshots. The spatial distribution of users is sampled from a correlated distribution, that tends to yield clusters of users, using a similar approach to [16] which was introduced to generate spatially correlated shadow maps. The number of active users in the hotspot area during snapshot $s$ is then given by:

$$
N_{a u}(s)=\frac{N h_{f} P U_{a} l(s)}{A_{h} N_{o}}
$$




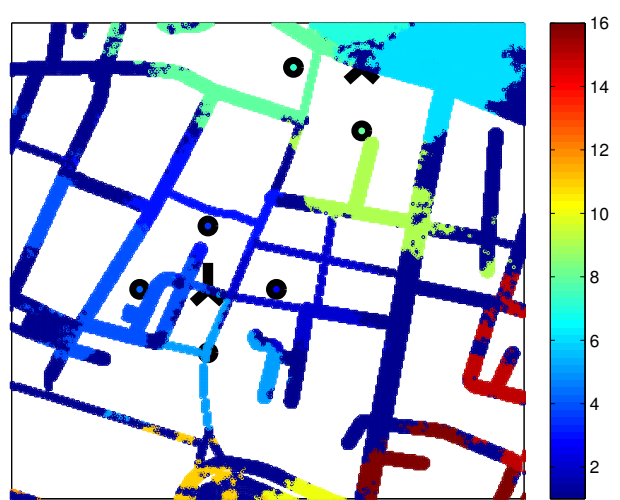

Fig. 7: Estimated serving cell where SCBSs are positioned in $40 \mathrm{~m}$ radius from the centre of each macro BS. Colour map indicates the cell ID, all macro BS are identified by ID 1 and SCBSs are numbers 2 to 17. Black 3-sectors icon and small coloured-circles represent the MBS and SCBS respectively.

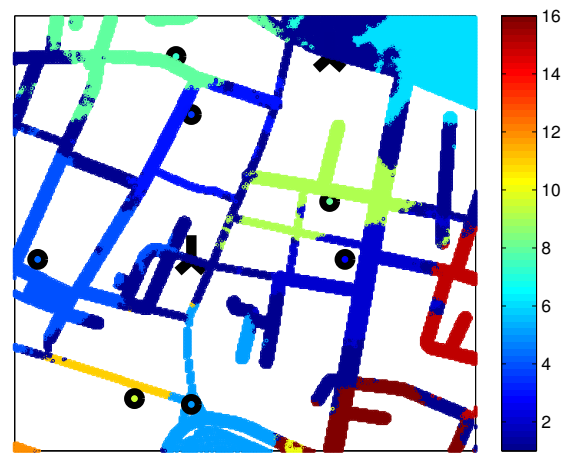

Fig. 8: Estimated serving cell where SCBSs are positioned in $100 \mathrm{~m}$ radius from the centre of each macro BS. Colour map indicates the cell ID, all macro BS are identified by ID 1 and SCBSs are numbers 2 to 17 . Black 3-sectors icon and small coloured-circles represent the MBS and SCBS respectively.

where $A_{h}$ is the area of the hotspot, $h_{f}$ a scaling factor, $P U_{a}$ the probability of the user being active, and $l(t)$ is a normalised load factor. The number of active users in each macro cell is obtained similarly. For the purpose of this work, we only focus on the performance of the outdoor users affected by the outdoor SCBS. We used the models in [18] to estimate the power consumptions of the SCBSs. Power consumptions are measured over 1 day user activity period. Figure 11 illustrates the $k W h$ power consumption of three different idle mode selection procedures i.e.

1) Network-assisted idle mode selection with perfect esti-

TABLE III: User traffic characteristics

\begin{tabular}{|l|l|}
\hline User Traffic parameter & Value \\
\hline Number of users per $K \mathrm{Km}^{2}(N)$ & 6000 \\
\hline Hotspot factor $\left(h_{f}\right)$ & 6 \\
\hline Operator market share $\left(N_{o}\right)$ & 0.4 \\
\hline Voice activity per month & 512 mins \\
\hline Data activity per month & $850 \mathrm{MB}$ \\
\hline Mean throughput activity & $330 \mathrm{Kbps}$ \\
\hline
\end{tabular}

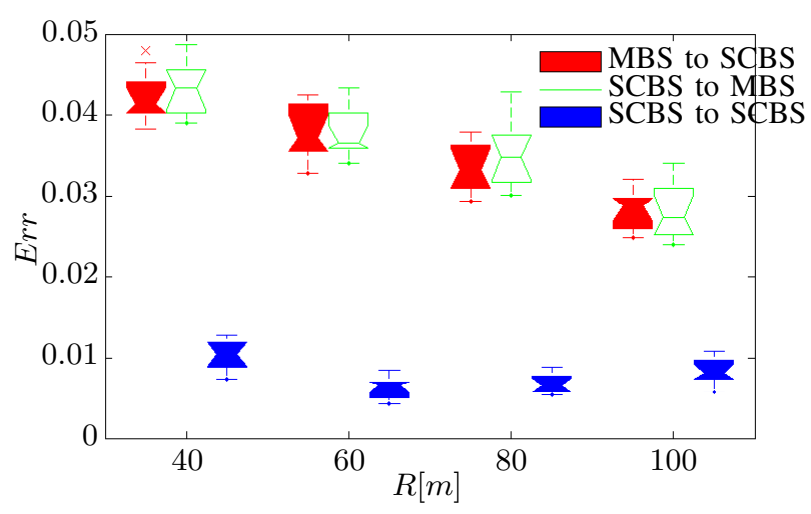

Fig. 9: Misclassification error of Classifier (b) with full data size and various radius's $(R)$ of the SCBS from the centre of each Macro BS.

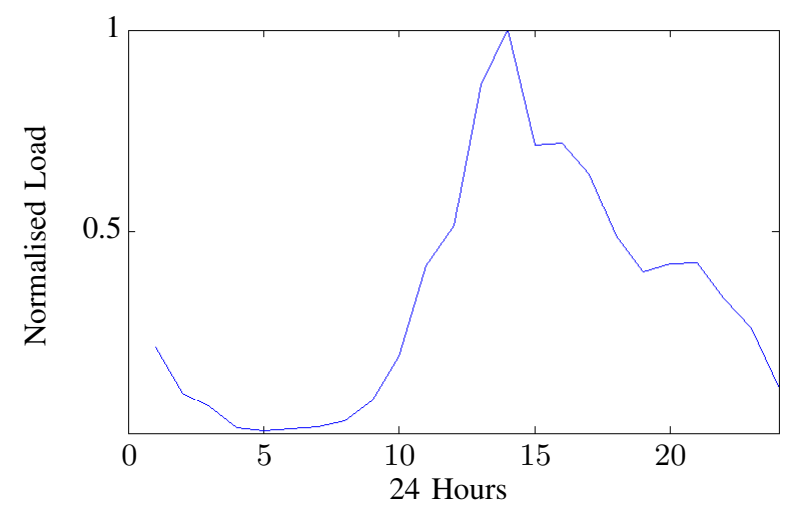

Fig. 10: Normalised daily traffic demand [17]

mation where misclassification error is zero.

2) Network-assisted idle mode selection using classifier (b)

3) Sniffer-based waking-up of the SCBSs [10] where SCBSs monitor the uplink received power from the users and wake up if the received power is larger than a certain threshold which is set to $-60 \mathrm{dBm}$ in this work.

Observe that for readability reasons we have not included power consumption values when no idle mode procedure is in place. In fact concurrent operation of all SCBSs in the scenario accounts for $2.07 \mathrm{kWh}$ power consumption. While Networkassisted idle mode selection using classifier (b) introduces an average of $0.15 \mathrm{kWh}$ power consumption. This can be translated into significant energy savings i.e. approximately $90 \%$. It can be observed that erroneous misclassification results in better energy efficiency gains, this is due to the fact that a larger percentage of misclassification is categorised as misclassifying a SCBS user as a MBS user. As expected up-link power monitoring methods result in lower energy efficiency gains due to the lack of communication between the cells. Moreover, Fig 12 shows that the user's throughput distribution not only is sacrificed by the adaptive selection of the small cells but also it has been improved compared with the simultaneous operation of all the SCBSs. This adaptive selection based on the network load reduces inter and intra layer interference and 


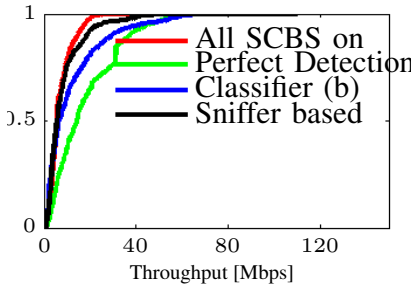

(a) $\mathrm{R}=40 \mathrm{~m}$

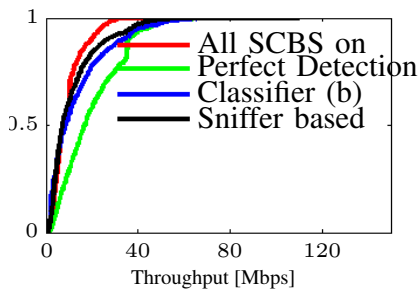

(b) $\mathrm{R}=60 \mathrm{~m}$

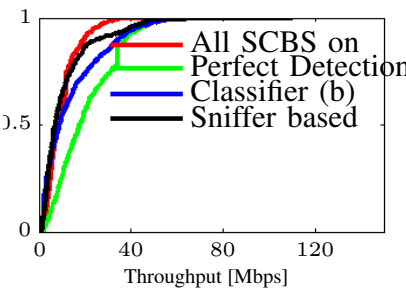

(c) $\mathrm{R}=80 \mathrm{~m}$

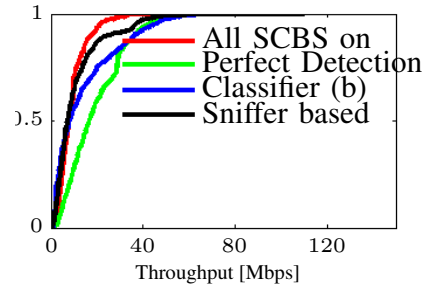

(d) $\mathrm{R}=100 \mathrm{~m}$

Fig. 12: CDF of the user throughputs for various idle mode procedures when 4 SCBSs are position on a circle of radius $R$ from the centre of each macro BS.

hence improves the received signal quality.

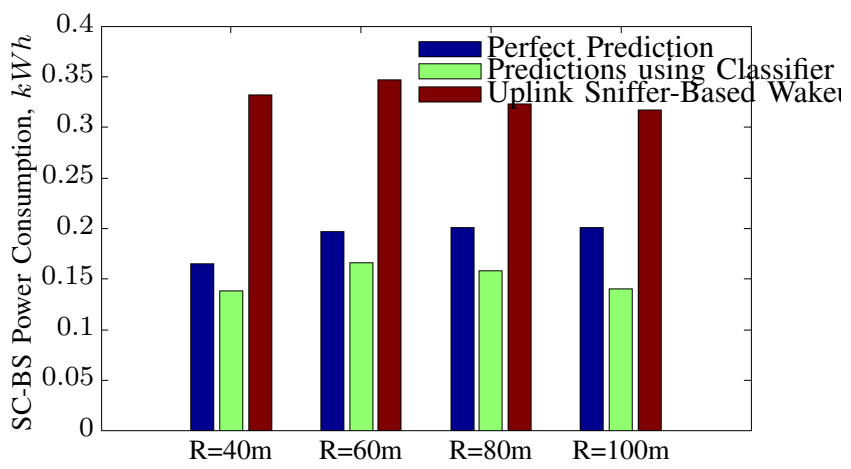

Fig. 11: Power Consumption, kWh

\section{CONCLUSiOnS}

In this paper we exploit records of the users' RF fingerprint vectors to estimate their serving cells. We introduce the use of a number of classifiers and we show that an average $1.9 \%$ misclassification error rate is achievable when the proposed two stage classifier is applied on the field measurements. We evaluate our method for various network configurations and various density of the small cells. And we have shown that by using this approach and taking advantage of the traffic load variations, energy efficiency gains as large as $90 \%$ are achievable compared to a configuration without idle modes for a given realistic network configurations.

\section{REFERENCES}

[1] C. V. N. Index, "Global mobile data traffic forecast update, 2014-2019," Cisco white paper, 2015.

[2] J. G. Andrews, H. Claussen, M. Dohler, S. Rangan, and M. C. Reed, "Femtocells: Past, present, and future," Selected Areas in Communications, IEEE Journal on, vol. 30, no. 3, pp. 497-508, 2012.

[3] R. Razavi and H. Claussen, "Urban small cell deployments: Impact on the network energy consumption," in Wireless Communications and Networking Conference Workshops (WCNCW), 2012 IEEE, pp. 47-52, IEEE, 2012.

[4] A. Mesodiakaki, F. Adelantado, A. Antonopoulos, E. Kartsakli, L. Alonso, and C. Verikoukis, "Energy impact of outdoor small cell backhaul in green heterogeneous networks," in IEEE CAMAD 2014 IEEE, DOI: 10.13140/2.1.3618.0162, 2014.
[5] J. T. Louhi, "Energy efficiency of modern cellular base stations," in Telecommunications Energy Conference, 2007. INTELEC 2007. 29th International, pp. 475-476, IEEE, 2007.

[6] I. Ashraf, F. Boccardi, and L. Ho, "Sleep mode techniques for small cell deployments," Communications Magazine, IEEE, vol. 49, no. 8, pp. 72-79, 2011

[7] R1-104968, Summary of the Description of Candidate eICIC Solutions, $3 G P P$ Std, Madrid, Spain, Aug. 2010.

[8] D. Lopez-Perez, I. Guvenc, G. De La Roche, M. Kountouris, T. Q. Quek, and J. Zhang, "Enhanced intercell interference coordination challenges in heterogeneous networks," Wireless Communications, IEEE, vol. 18, no. 3, pp. 22-30, 2011.

[9] B. Partov, D. Leith, and R. Razavi, "Energy aware configuration of small cell networks," in To be appeared in IEEE International Symposium on Personal, Indoor and Mobile Radio Communications (PIMRC), IEEE 2014.

[10] H. Claussen, I. Ashraf, and L. T. Ho, "Dynamic idle mode procedures for femtocells," Bell Labs Technical Journal, vol. 15, no. 2, pp. 95-116, 2010.

[11] ETSI TR 136927 V11.0.0 (2012-10) LTE, Evolved Universal Terrestrial Radio Access (E-UTRA), Potential solutions for energy saving for EUTRAN (3GPP TR 36.927 version 11.0.0 Release 11), 2012.

[12] 3rd Generation Partnership Project; Technical Specification Group Radio Access Network; Evolved Universal Terrestrial Radio Access (E-UTRA); Further advancements for E-UTRA physical layer aspects (Release 9), 2010.

[13] https://github.com/andr3jx/Mobilog.

[14] T. Hastie, R. Tibshirani, J. Friedman, T. Hastie, J. Friedman, and R. Tibshirani, The elements of statistical learning, vol. 2. Springer, 2009.

[15] http://www.askcomreg.ie/mobile/siteviewer.273.LE.asp.

[16] H. Claussen, "Efficient modelling of channel maps with correlated shadow fading in mobile radio systems," in Personal, Indoor and Mobile Radio Communications, 2005. PIMRC 2005. IEEE 16th International Symposium on, vol. 1, pp. 512-516, IEEE, 2005.

[17] E. Carolan, S. C. McLoone, S. F. McLoone, and R. Farrell, "Analysing ireland's interurban communication network using call data records.," in 23rd Irish Signals and Systems Conference, June 28-29 2012.

[18] G. Auer, O. Blume, V. Giannini, I. Godor, M. Imran, Y. Jading, E. Katranaras, M. Olsson, D. Sabella, P. Skillermark, et al., "D2. 3: energy efficiency analysis of the reference systems, areas of improvements and target breakdown," INFSOICT-247733 EARTH (Energy Aware Radio and NeTwork TecHnologies), Tech. Rep, 2010. 